\title{
Significance of Phi bodies in acute leukaemia
}

\author{
L DE SALVO CARDULlo, R MORILlA, AND D CATOVSKY
}

From the MRC Leukaemia Unit, Royal Postgraduate Medical School, Ducane Road, London W12 OHS,UK

SUMMARY Material from 39 patients with acute leukaemia was investigated with the peroxidase cytochemical reaction using 3,3'diaminobenzidine (DAB) and other substrates in order to test their sensitivity in detecting myeloid differentiation. The proportion of positive blasts and of cases with Auer rods in acute myeloid leukaemia (AML) was significantly greater with DAB than with benzidine. In addition, Phi bodies were demonstrated in AML blasts only when DAB was used; Phi bodies were also observed in two out of seven cases of chronic granulocytic leukaemia in 'myeloid' blast crisis but were not seen in any case of acute lymphoblastic leukaemia. Phi bodies were more numerous when the reaction was carried out at $\mathrm{pH} 9 \cdot 7$, and their number was significantly reduced in the presence of 3-amino 1,2,4-triazole. Both findings suggest that the Phi bodies derive from catalase-containing granules (microperoxisomes) and are distinct from Auer rods, which derive from peroxidase-containing (primary) granules. Like Auer rods, Phi bodies appear to be characteristic of immature myeloid cells in leukaemia but are seen with a higher frequency than Auer rods in acute myeloid leukaemia.

The cytochemical reaction for myeloperoxidase is a valuable tool in the classification of acute leukaemia, chiefly in the distinction between acute myeloid and acute lymphoblastic leukaemia. ${ }^{1}$

Auer rods ${ }^{2}$ are usually visible with Romanovsky stains in blast cells from various acute myeloid types, particularly those with myeloblastic-promyelocytic differentiation (M1 to M3 of the FAB classification). ${ }^{1}$ They have been considered to result from aberrations of the primary (azurophil) granules of early granulocytic cells ${ }^{3}$ and are often seen more easily in peroxidase preparations. Hanker et al. ${ }^{4}$ recently described a new type of rod, the Phi body, not visualised with Romanovsky dyes but only by means of a peroxidase reaction using 3,3 'diaminobenzidine (DAB), and suggested that they were also characteristic of acute myeloid leukaemia.

We have investigated the presence of Auer rods and Phi bodies in a series of patients with acute leukaemia in order to clarify the relation of Auer rods to the so-called Phi bodies.

\section{Material and methods}

Bone marrow and peripheral blood films from 32 patients with acute leukaemia (16 myeloid and 16

Accepted for publication 30 July 1980 lymphoblastic) and seven with chronic granulocytic leukaemia in 'myeloid' blast crisis were studied. The leukaemia was classified according to the FAB criteria $^{1}$ using May-Grünwald-Giemsa stain and a battery of cytochemical reactions: Sudan Black B, naphthol-AS acetate esterase $\pm \mathrm{NaF}, \alpha$-naphthyl acetate esterase $\pm \mathrm{NaF}$, acid phosphatase, and lysozyme.

The myeloperoxidase reaction was investigated with the following reagents: (1) benzidine dihydrochloride; (2) 2,7, fluorenediamine; and (3) 3,3'diaminobenzidine tetrahydrochloride (DAB), introduced by Graham and Karnovsky in $1966 .{ }^{5}$ Substrates (1) and (2) were used according to previously published techniques. ${ }^{6}$ DAB was tested according to the simplified technique of Hanker et al. ${ }^{7}$ with minor modifications, as follows:

FIXATION

One minute in a mixture of $1.25 \%$ glutaraldehyde and $1 \%$ formaldehyde in $0.1 \mathrm{M}$ phosphate buffer (pH 7.3); rinsing in $0.9 \% \mathrm{NaCl}$.

INCUBATION

One minute in a medium (prepared just before use) containing $5 \mathrm{mg} \mathrm{DAB}, 10 \mathrm{ml}$ Tris $\mathrm{HCl}$ buffer $\left(0.05 \mathrm{M}, \mathrm{pH} 7.6\right.$ ), and $0.1 \mathrm{ml} 1 \% \mathrm{H}_{2} \mathrm{O}_{2}$. Slides are then rinsed briefly in Tris- $\mathrm{HCl}$ buffer and immersed 
for 1 minute in $0.5 \% \mathrm{CuSO}_{4}$ in Tris- $\mathrm{HCl}$ buffer and rinsed again in $0.9 \% \mathrm{NaCl}$.

\section{COUNTERSTAIN}

Ten minutes in a $10 \%$ solution of Giemsa; mounted in DPX.

In several samples the reaction with DAB was also

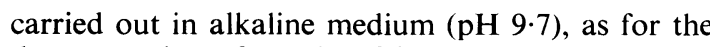
demonstration of catalase,$^{8}{ }^{9}$ with and without the addition of 3-amino 1,2,4-triazole (AMT), a catalase inhibitor. ${ }^{10}$ AMT was used at a concentration of $2 \times 10^{-2} \mathrm{~mol} / \mathrm{l}$ for 30 minutes at $4^{\circ} \mathrm{C}$ before the cytochemical reaction and was also included in the incubation mixture of the reaction.
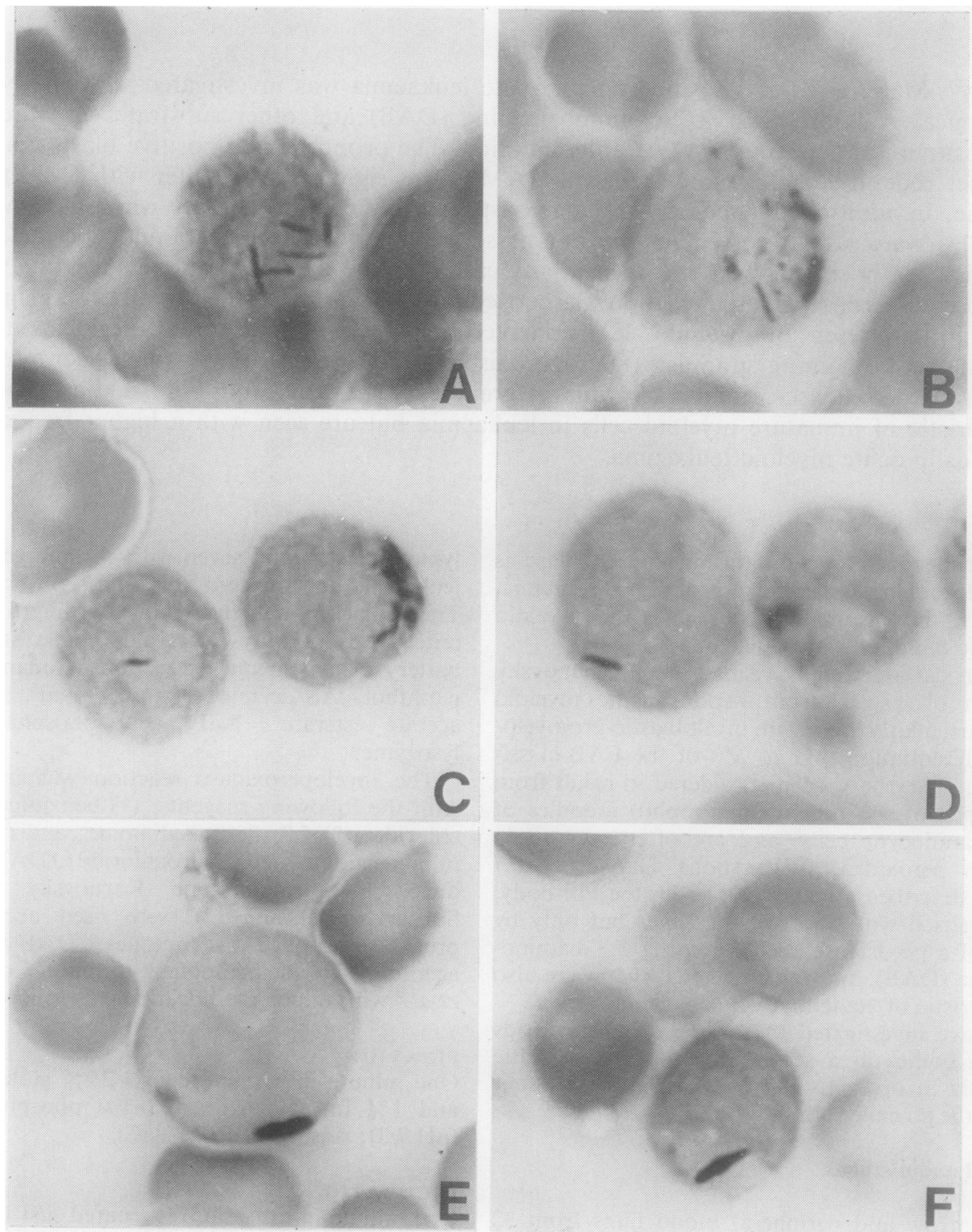

Blast cells of $A M L$ cases showing Phi bodies $(A, B, C, D)$ or Auer rods $(E, F)$. Phi bodies were seen as thin, sometimes multiple $(A)$, rods, while Auer rods were often single and thicker $(E, F)$ than Phi bodies. (Peroxidase reaction with DAB $\times 1400$ ). 


\section{Results}

The cases of acute myeloid leukaemia (AML) and chronic granulocytic leukaemia in 'myeloid' blast crisis (CGL-BC) were considered together for the analysis of peroxidase activity. As the results with substrates (1) and (2) were very similar, ${ }^{6}$ only the comparison of benzidine hydrochloride with DAB will be described here.

Three features were compared: overall reactivity (that is, the percentage of positive blast cells), and the incidence of Auer rods and Phi bodies. The latter were visualised only with DAB (Fig. 1A-E) and were single or multiple (Fig. 1A), fusiform, thin rods often easily distinguishable from the relatively thicker Auer rods (Fig. 1E,F). The distinction between the two types of rods was not always possible, and for some of the comparisons we have scored the incidence of both rods together.

Table 1 shows the results with both substrates in all the cases studied. Both reactions were positive in variable degree in all AML and CGL-BC cases. The proportion of blasts with a positive cytoplasmic reaction was, in each type, greater when $D A B$ was used $(\mathrm{P}<0.001$, paired Student's $t$ test). In one case of M5 leukaemia, $11 \%$ of positive blasts were seen only with DAB. The most striking difference was observed in the incidence of rods. Auer rods were detected three times more often with DAB ( $P<0.02$, Fischer's exact test), and Phi bodies were seen in over two-thirds of cases $(P<0.001$, Fischer's exact test), particularly in several in which no Auer rods were detected by either substrate, and they were not visible in the Romanovsky-stained films. The cases with Phi bodies included two out of seven with CGL-BC. Phi bodies were equally frequent in M1M2 and M4-M5 cases; Auer rods were slightly more common in M1-M2. The proportion of blast cells with Phi bodies ranged from $<1 \%$ to $15 \%$ in the positive cases; Auer rods ranged from $1 \%$ to $8 \%$. None of the cases of acute lymphocytic leukaemia
Table 2 Percentage of Phi bodies and Auer rods at two $p H s$ with $D A B$

\begin{tabular}{lcc}
\hline Diagnosis & $p H 7 \cdot 6$ & $p H 9 \cdot 7$ \\
\hline AML (M1) & 18 & 26 \\
AML (M1) & 11 & 24 \\
AML (M1) & 19 & 30 \\
AML (M2) & 14 & 17 \\
AML (M4) & 3 & $9 \cdot 5$ \\
AML (M4) & 7 & 7 \\
AML (M5) & 2 & 13 \\
CGL-BC (M1) & $<1$ & 8 \\
CGL-BC (M5) & 0 & $1 \cdot 2$ \\
CGL-BC (M5) & $<1$ & 11 \\
\hline Mean & $7.6 \% *$ & $14.7 \% *$
\end{tabular}

*Difference between means was statistically significant $(P<0.001$, paired Student's $t$ test).

Table 3 Effect of aminotriazole (AMT) in the percentage of Phi bodies and Auer rods

\begin{tabular}{lrll}
\hline Diagnosis & pH 7.6 & $\begin{array}{l}\text { pH } 9.7 \\
\text { without } \text { AMT }\end{array}$ & $\begin{array}{l}\text { pH } 9.7 \\
\text { with } A M T\end{array}$ \\
\hline AML (M1) & 29 & 44 & 9 \\
AML (M1) & 3 & 8 & 0 \\
AML (M1) & 19 & 30 & 2 \\
AML (M4) & 3 & 7 & 0 \\
\hline
\end{tabular}

tested showed positivity in blasts or the presence of rods with either substrate (Table 1).

The percentage of blast cells with rods at two different levels of $\mathrm{pH}$ was tested in 10 cases (Table 2). In nearly every instance, a higher proportion of leukaemic cells with rods, particularly Phi bodies, was seen at the more alkaline $\mathrm{pH}$; overall, twice as many cells were positive at $\mathrm{pH} 9.7(\mathrm{P}<0.001$; Student's $t$-test, paired samples). In the three cases of CGL-BC tested, this difference appeared more striking as very few positive cells were seen at $\mathrm{pH}$ $7 \cdot 6$ while positive blasts were readily seen at $\mathrm{pH} 9 \cdot 7$. The overall proportion of blasts with positive granules, not rods, was, in contrast, lower at $\mathrm{pH} 9.7$ (data not shown).

In four cases, the DAB reaction at $\mathrm{pH} 9 \cdot 7$ was

Table 1 Comparison of results with both substrates

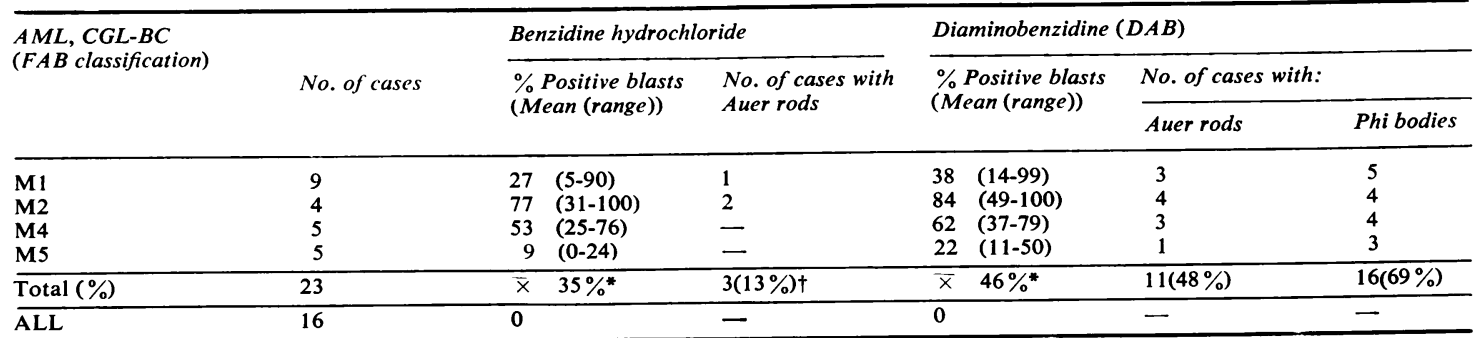

*Difference between means was statistically significant ( $P<0.001$, paired Student's $t$ test).

"Difference in incidence of Auer rods was significant $(P<0.02$, Fischer's exact test). The difference between the incidence of Phi bodies (with DAB) and Auer rods (with benzidine hydrochloride) was also statistically significant $(P<0 \cdot 001$, Fischer's exact test).

$A L L=$ acute lymphocytic leukaemia. 
tested in the presence of AMT (Table 3). An almost complete inhibition of the reaction in rods was observed. The reaction in the cytoplasm of blasts did not change with AMT.

\section{Discussion}

We have confirmed the observation of Hanker et al. 4 that in a high proportion of AML cases spindle or fusiform shaped rods (Phi bodies) occur with a greater frequency than Auer rods when DAB is used as substrate for the peroxidase reaction. In addition, we have shown Phi bodies in two out of seven cases of CGL-BC. Because DAB is not specific for myeloperoxidase and can also demonstrate catalase activity, ${ }^{8}$ Hanker designated the cytochemical reaction he described as hydroxyperoxidase. ${ }^{4}$

The use of DAB reveals Phi bodies and is also a very sensitive method for demonstrating peroxidase activity in leukaemic blast cells (Table 1). We therefore now use DAB routinely in preference to the other substrates, benzidine and 2,7, fluorenediamine. DAB is also used for the demonstration of myeloperoxidase activity at electron microscopy level..$^{510} \mathrm{It}$ is possible, therefore, that one of the reasons for the greater sensitivity of the electron microscopy technique results from the use of DAB and not only from the higher resolution of the system. This is important because when comparisons of the value of electron microscopy techniques are made, the type of substrate used at light microscopy is rarely taken into consideration. One of our M5 cases showed peroxidase activity only with DAB while the reaction was negative with benzidine hydrochloride.

Of great interest is the relation of Phi bodies to Auer rods, partly discussed by Hanker et al. ${ }^{7}$ Using the technique with DAB, it is sometimes difficult to distinguish them because both are strongly positive with this reagent. The experiments carried out at pH 9.7 and the inhibition by AMT, a known catalase inhibitor, support the suggestion that Phi bodies may originate from catalase-containing

Table 4 Possible explanation for the higher reactivity with the HPO reaction

\begin{tabular}{lll}
\hline Granules (rods) & \multicolumn{2}{l}{ Substrate used } \\
\cline { 2 - 3 } & Benzidine HC & DAB \\
\hline $\begin{array}{l}\text { Primary, MPO positive } \\
\text { (Auer rods) }\end{array}$ & + & + \\
$\begin{array}{l}\text { Microperoxisomes, catalase-positive } \\
\text { (Phi bodies) }\end{array}$ & - & + \\
\hline HPO = hydroperoxidased & & + \\
MPO = myeloperoxidase &
\end{tabular}

granules. ${ }^{4}$ As shown in Table 4, the greater sensitivity of the hydroperoxidase reaction can therefore be explained on a more simple basis. The conventional substrates used for the myeloperoxidase reaction (eg, benzidine hydrochloride) demonstrate enzyme activity in the primary granules, which are known to fuse into Auer rods in AML cells. ${ }^{3}$ The inhibition with AMT, and particularly the enhancement observed when the reaction was tested in the more alkaline medium, suggests that Phi bodies may be comparable to Auer rods in that they are specific to leukaemic cells but are derived from catalasecontaining granules, the microperoxisomes of Novikoff et al. ${ }^{8}$ (Table 4). These granules were demonstrated at electron microscopic level in human promyelocytes and promonocytes by means of DAB at $\mathrm{pH} 9 \cdot \mathbf{7 . 9}$

Despite the higher incidence of Phi bodies at $\mathrm{pH}$ 9.7, we recommend the routine testing of leukaemic samples with DAB at pH 7.6 because the overall cytoplasmic reaction, largely due to myeloperoxidase, is greater, and, in most cases, Phi bodies are also clearly visualised. In special cases, when the main interest is the demonstration of Phi bodies, the reaction at $\mathrm{pH} 9.7$ should also be performed. This may be the case when attempts are made to identify the various blast cell populations in CGL-BC, especially as Phi bodies are not seen in lymphoblasts.

In conclusion, we have extended the studies by Hanker et al.4 7 with the hydroperoxidase cytochemical reaction, which permits the demonstration of Phi bodies in most AML and some CGL-BC cases. We have also provided new evidence supporting the origin of those rods from catalase-containing granules. More work is necessary, particularly at electron microscopic level with DAB in the more alkaline medium, to clarify the morphology of the Phi bodies and their relation to the traditional Auer rods.

$\mathbf{L}$ de Salvo Cardullo was in receipt of a scholarship from the Banco de Sangre (Edo de Zulia), Maracaibo, Venezuela.

References

${ }^{1}$ Bennett JM, Catovsky D, Daniel MT, Flandrin G, N Galton DAG, Gralnick HR, Sultan C. Proposals for the N classification of the acute leukaemias. (FAB Co- $\omega$ operative Group). Br J Haematol 1976;33:451-8.

2 Auer J. Some hitherto undescribed structures found in the 0 large lymphocytes of a case of acute leukaemia. $\mathrm{Am} J \stackrel{\mathrm{D}}{\mathrm{D}}$ Med Sci 1906;131:1002-15.

Bainton DF, Friedlander LM, Shohet SB. Abnormalities in granule formation in acute myelogenous leukaemia. Blood 1977;49:693-704.

- Hanker JS, Laszlo J, Moore JG. The light microscopic demonstration of hydroperoxidase-positive Phi bodies and rods in leukocytes in acute myeloid leukaemia. 
Histochem $1978 ; 58: 241-52$.

- Graham RC, Karnovsky JM. The early stages of absorption of injected horse-radish peroxidase in the proximal tubules of mouse kidney; ultra-structural cytochemistry by a new technique. J Histochem Cytochem 1966;14: 291-302.

- Benavides I, Catovsky D. Myeloperoxidase cytochemistry using 2,7-fluorenediamine. J Clin Pathol 1978;31:1114-6.

7 Hanker JS, Ambrose WW, James CJ, Mandelkorn J, Yates PE, Gall SA, Bossen EH, Fay JW, Laszlo J, Moore JO. Facilitated light microscopic cytochemical diagnosis of acute myelogenous leukaemia. Cancer Res 1979;39:1635-9.

${ }^{8}$ Novikoff AB, Novikoff PM, Davis C, Quintana M. Studies on microperoxisomes. II A cytochemical method for light and electron microscopy. $J$ Histochem Cytochem 20:1006-23.

- Breton-Gorius J, Guichard J. Cytochemical distinction between azurophils and catalase-containing granules in leukocytes: distribution in human promyelocytes and promonocytes. J Reticuloendothel Soc 1978;24:637-46.

10 Breton-Gorius J, Coquin Y, Guichard J. Cytochemical distinction between azurophils and catalase-containing granules in leukocytes. Lab Invest 1978;38:21-31.

Requests for reprints to: Dr D Catovsky MRC Leukaemia Unit, Royal Postgraduate Medical School, London W12 0HS. 